\title{
Probing the nuclear matter isospin asymmetry by nucleon-induced reactions at Fermi energies
}

\author{
F. Sébille ${ }^{1}$, V. de la Mota ${ }^{1}$ \\ ${ }^{1}$ SUBATECH, Université de Nantes, CNRS/IN2P3, Nantes, France \\ I.C. Sagrado Garcia ${ }^{2}$, J.F. Lecolley ${ }^{2}$ \\ ${ }^{2}$ LPC, ENSICAEN, Université de Caen, CNRS/IN2P3, Caen, France \\ V. Blideanu ${ }^{3}$, \\ ${ }^{3}$ CEA, CEN Saclay 91191 Gif-sur-Yvette
}

\begin{abstract}
Despite the fact that valuable experimental measures are still lacking, available nuclear data on nucleon-induced reactions open new opportunities to address either reaction mechanisms or nuclear interaction characteristics. In this work single and double differential cross sections of emitted particles are analyzed and compared with the experiment. It will be evidenced that these cross sections follow a precise hierarchy. The pre-equilibrium components of the spectra are built up by the dynamics of the reaction as well as by the properties of the nuclear interaction.
\end{abstract}




\section{Introduction}

Even though an exhaustive experimental systematics in nucleon-induced reactions is far from being achieved at intermediate energies, the available nuclear data [1]-[5] open new opportunities to the theoretical models which aim at addressing either reaction mechanisms or nuclear interaction characteristics. The theoretical description [6] of these processes with a microscopic approach constitutes a difficult task. One difficulty is the treatment of composite particle emission, which in most models, gives unrealistic results. Nevertheless at intermediate energies, the production rates of light charged particles are important, and require a relevant evaluation, even if we are mainly interested by nucleonic probes in the incoming and outgoing channels. In this work we investigate nucleon-induced reactions in the framework of the Dynamical Wavelets in Nuclei (DYWAN) model, which has been proven to give a good description of the nuclear dynamics in nucleon on nucleus [7] or in nucleus on nucleus [8] reactions. The purpose of the model is to tackle efficiently the out of equilibrium mechanisms contributing to the observables. In few words, the essential aspects of our model are the quantum treatment of nucleons in terms of Slater determinants of wavelets [9] which evolve through an ETDHF-type equation [10]. Density fluctuations, responsible of the break-up of the system, are generated by unfolding the averaged density on many slater determinants of single nucleons, each of which undertaking a particular path in the available space of states of the system.

In order to compare with the experiment and to avoid unnecessary time consuming calculations in this framework, when necessary, the evaporative component will be estimated on statistical grounds like for example with the GEMINI [11] evaporation code. Comparative theoretical-experimental investigations on heavy-ion collisions and on nucleon-induced reactions around the Fermi energy reveal [12]-[16] the importance of a proper inclusion of the isospin-dependent term in effective nuclear interactions. Theoretical and experimental results exhibit a conspicuous sensitivity to the nature of both the incoming and the detected nucleons. Those results suggested to go into thoroughly, in order to get more insight on the physics involved in these asymmetric nuclear reactions induced by nucleon beams. In this work we focus on nucleon differential crosss-sections, either simple or double, depending on the available nuclear data in nucleon-induced reactions, for different incident particles, at different incident energies and for different targets.

This work is organized as follows. A brief survey of the formalism is presented in Sec. 2. In Sec. 3 the influence of the isospin assymmetry part of the force is 
adressed. In Sec. 4 the effects of the overall mean-field and of the target mass on theoretical results is analyzed. A hierachy of emission cross sections is shown to emerge in Sec. 5. In Sec. 6 we summarize our results and conclude.

\section{Survey of the model}

The initial conditions of the nucleon-nucleus reactions starts from the self-consistent research of the target ground state, whose Hartree-Fock stationary one-body wave functions are evaluated according to a wavelet decomposition determined by a maximum entropy criterion:

$$
\left|\varphi^{\lambda}>=\sum_{i}^{N_{\lambda}} \omega_{i}^{\lambda}\right| \alpha_{i}^{\lambda}>
$$

Here $\left\{\mid \alpha_{i}^{\lambda}>\right\}$ is a spline wavelet basis [17], the quantities $\omega_{i}^{\lambda}$ are their corresponding weights and $N_{\lambda}$ is the number of wavelets in the expansion of the $\lambda^{t h}$ level wave function. In current simulations $N_{\lambda}$ depends on the concerned level, typically its average value is around 10 at the first level of scale decomposition. In this approach the incoming nucleon is described by a wavefunction in the transversal plane with respect to the incident beam. It is designed according to the average energy and width of the experimental nucleon beams. This wavefunction is expanded in a set of wavelets. The expansion keeps track of the impact parameters through a spatial paving made in terms of centred rings covering a large region . The geometric extension of this decomposition is larger than the corresponding transverse area of the target. Typically 200 to 300 wavelets are used in the incident wave decomposition in order to sample a large range of impact parameters from inelastic to elastic scattering. During the reaction the incident nucleonic wave is propagated and scattered by the target. There, different transport processes take place as a consequence of the interaction between both partners. The emission spectra will then keep the fingerprints of these microscopic processes.

The implemented basis is a particular case of generalized coherent states [15], which are characterized by their group properties. Accordingly, as shown in Ref. [10], the time evolution of their moments is governed by a set of coupled differential equations which can be derived from a variational principle. The essential information on the nuclear mean field is contained in the motion and spreading of wavelets. Two-body correlations, which are introduced by a master equation for the single-particle occupation numbers, are responsible of the transitions between different energy levels. The transition rates are calculated in the framework of the 
Born approximation, by using the free nucleon-nucleon elastic cross section, without in-medium effects.

The many-body density matrix of the system is given by a linear combination of Slater determinants $\mid \Phi^{N}>$ of single particle wave functions. Due to the orthonormality properties of the wavelets and in virtue of Eq. (1), each $\left|\Phi^{N}\right\rangle$ can be written as a linear combination of Slater determinants of wavelets:

$$
\left|\Theta_{M}(t)>=\right| \alpha_{i_{1}}^{\lambda_{1}}>\wedge \ldots \wedge \mid \alpha_{i_{N}}^{\lambda_{N}}>
$$

$M$ standing for a given set of indices $\left\{i_{1}, \ldots, i_{N}\right\}$. It means that for a system of $\mathrm{N}$ identical particles, the Slater determinant is built from $\mathrm{N}$ wavelets. As shown elsewhere [18], the associated least biased N-body density operator can be then expressed as a superposition of a fixed number of density operators related to wavelet Slater determinants, according to the expression:

$$
\left.\left|D^{N}(t)>=\sum_{K}^{N_{K}}\right| a_{K}(t)\right|^{2} \sum_{M_{K}, M_{K}^{\prime}} b_{M_{K}} b_{M_{K}^{\prime}}^{*}\left|\Theta_{M_{K}}><\Theta_{M_{K}^{\prime}}\right|
$$

where $a_{K}(t)$ are the weights of single-particle Slater determinants and $b_{M_{K}}$ are the coefficients of Slater determinants of wavelets coming from the wavelet decomposition. In order to span as well as possible the set of accessible states, $N_{K}$ ranges from $10^{5}$ to $10^{6}$, which allows a good compromise between a reasonable computing time and a good accuracy.

Slater determinants describe the fluctuations around the mean behavior given by the one-body density matrix. Nevertheless, in the present work we will not address the question of the observable fluctuations since only comparisons with inclusive experimental results will be possible. Therefore, the chosen values of $N_{K}$ were taken to constrain the numerical uncertainties on the one-body observables to be less than 5 per cent.

The formation of clusters is based on wavelets overlap criteria [18]. This means that clusters are determined by the topology of phase space induced by the dynamics of the reaction, carrying essentially the fingerprints of mean-field fluctuations. The asymptotic conditions of fragments are attained by a classical coulombian trajectory prolongation at $500 \mathrm{fm} / \mathrm{c}$. At this time the individual collisions have mostly been suppressed, pre-equilibrium particles have already been emitted and the statistical emission, characteristic of the equilibrium stage, is started. For times greater than this "freeze-out" time the cluster structure of the already emitted particles remains invariant. This is due to the quantum spreading of the wavefunctions which acts 
to preserve their corresponding overlap. The remaining contribution related to the equilibrium statistical emission is deduced from the excitation energy stored in the heavy residue, which obviously decreases with greater limiting times for the dynamical calculations. For more details about the model we refer the reader to Ref. $[8,10]$.

\section{$3 \quad$ Isospin asymmetry fingerprints on emission cross sections}

Different experiments in nucleon-induced reactions on lead targets have been previously investigated in the Fermi energy domain either using neutrons [2, 3] or using protons [4] as incident projectiles. Let us first address ${ }^{208} \mathrm{~Pb}(\mathrm{n}, \mathrm{Xp})$ and ${ }^{208} \mathrm{~Pb}(\mathrm{p}, \mathrm{Xp})$ reactions at, respectively, $96 \mathrm{MeV}$ and $63 \mathrm{MeV}$ incident energies.

The experimental data [3] for the former are displayed with diamonds in Fig. 1a and in Fig. 1b. Those corresponding to the latter reaction [4] are shown in Fig. 1c with crosses. In Fig. 1b are also displayed the experimental results of the ${ }^{208} \mathrm{Bi}(\mathrm{n}, \mathrm{Xp})$ reaction at $63 \mathrm{MeV}$ [5] (crosses). Let us underline that they are close to the ${ }^{208} \mathrm{~Pb}(\mathrm{n}, \mathrm{Xp})$ results of Ref. [2]. We can remark that the results for the $\mathrm{Bi}$ target are in agreement with those of Fig. 1c, even if the target is different. It is well known that the differential cross sections vary smoothly with the target mass.

Comparing the experimental data from references [3] and [5], in Fig. 1b, we observe that these spectra exhibit very similar shapes and magnitudes. For both targets the maxima are lying close to $10 \mathrm{mb} / \mathrm{MeV}$. This underlines the fact that they are rather insensitive to the increase of the incident energy. In Fig. 1a and Fig. 1c the results of the DYWAN model are drawn with two implementations of a local Skyrme interaction [19], with (solid line) or without (dotted line) the isospin asymmetry contribution [20] to the effective nuclear interaction. Whether the isospin symmetry energy is switch on or not, it can be seen in Fig. 1a, that theoretical and experimental results for protons are close to each other.

In Fig. 1c, a clear difference appears between the two calculations concerning the proton spectra. As a matter of fact, the calculations taking into account the isospin asymmetry, point out a subtantial and important increase of the cross section magnitude when the projectiles are also protons. The inverse could have been expected, if we refer to reaction mechanism pictures where direct nucleon-nucleon diffusions are considered as dominant. It stems from the fact that the free nucleon-nucleon 

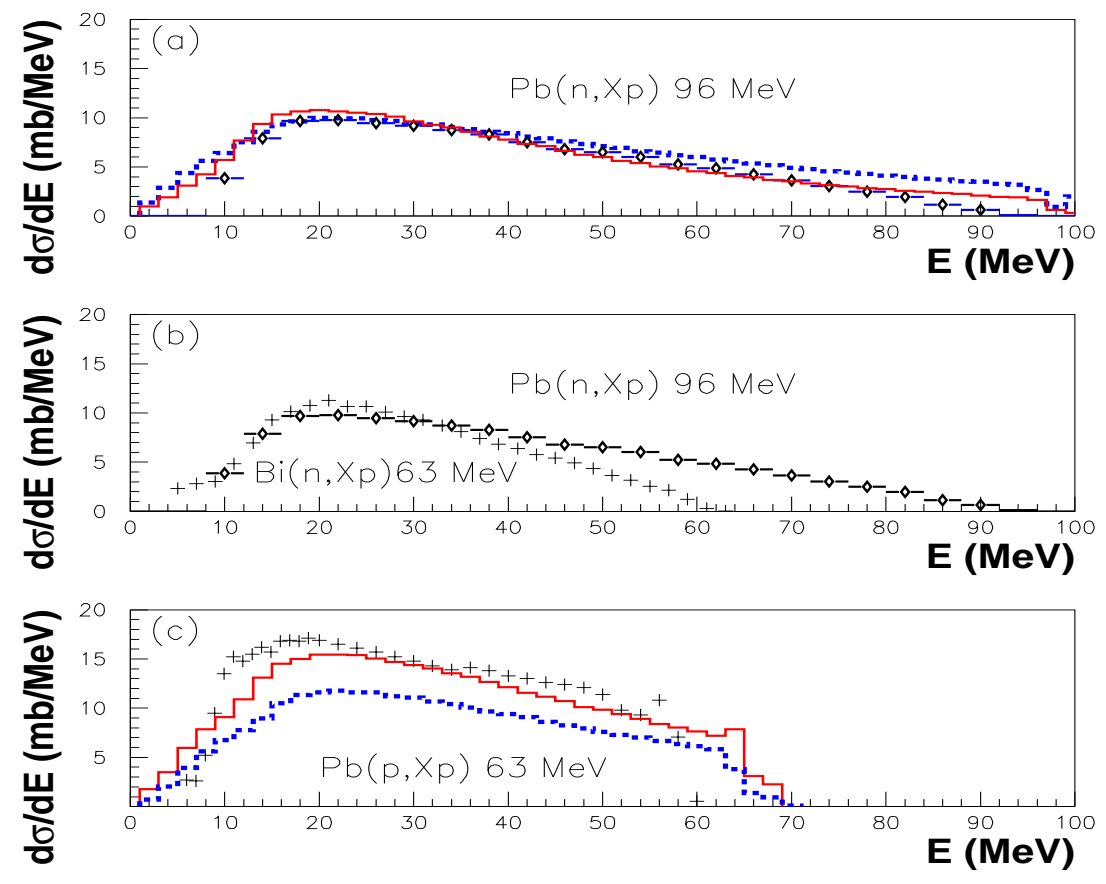

Figure 1: (Color online) (a) Energy differential cross sections in ${ }^{208} \mathrm{~Pb}(\mathrm{n}, \mathrm{Xp})$ reaction at $96 \mathrm{MeV}$. Experimental results (diamonds) are from Ref. [3]. Theoretical calculations are represented with solid line(dashed line) for the soft EOS with (without) asymmetry term. (b) Comparison between experimental differential cross sections related to ${ }^{208} \mathrm{~Pb}(\mathrm{n}, \mathrm{Xp})$ reaction at $96 \mathrm{MeV}$ from Ref. [3] (diamonds) and ${ }^{208} \mathrm{Bi}(\mathrm{n}, \mathrm{Xp})$ reaction at $63 \mathrm{MeV}$ from Ref. [5] (crosses). (c) Energy differential cross sections in ${ }^{208} \mathrm{~Pb}(\mathrm{p}, \mathrm{Xp})$ reaction at $63 \mathrm{MeV}$. Experimental results (crosses) are from Ref. [4]. Theoretical calculations use the same conventions as in Fig. 1a. 
diffusion cross sections [21,22] are conspicuously higher when the nucleon isospin degree of freedom of the two interacting particles is different. This inversion is observed in both theoretical and experimental results. The results of Fig. 1c suggest that, in the range of Fermi energies, the mean-field can produce strong effects and counterbalance direct diffusion processes. The sensitivity of the theoretical calculations to the isospin symmetry energy endorses the role of the mean-field behavior, since clear fingerprints appear only when the isospin of both the emitted nucleon and projectile are the same.

\section{Evidence of mean-field effects and mass depen- dence}
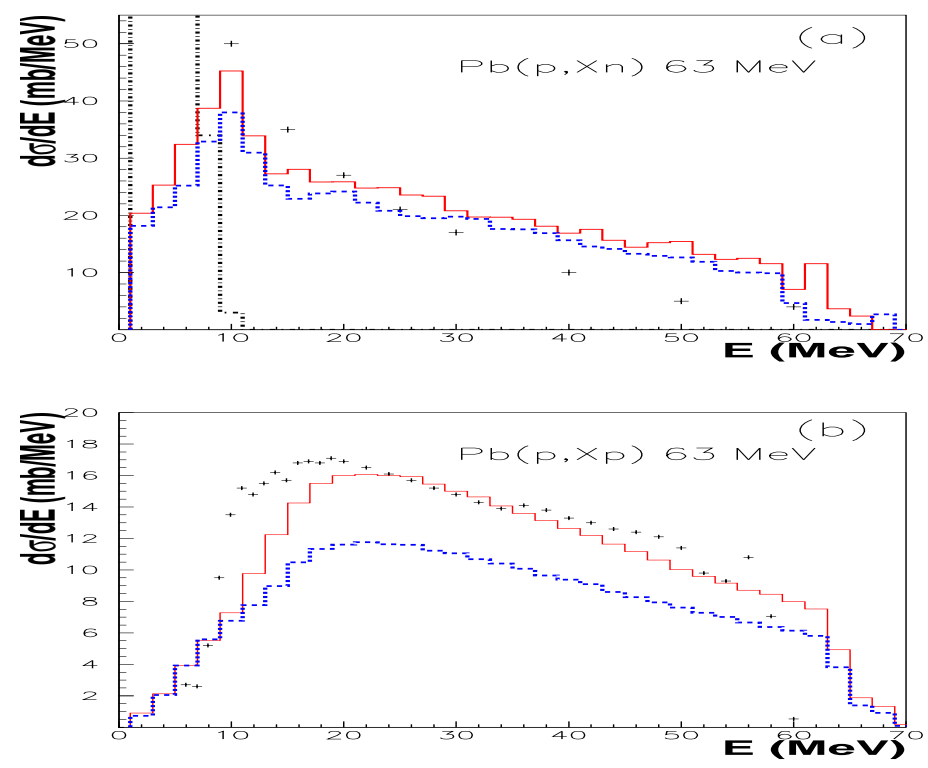

Figure 2: (Color online) (a) Differential cross sections in ${ }^{208} \mathrm{~Pb}(\mathrm{p}, \mathrm{Xn})$ reaction at $63 \mathrm{MeV}$. Experimental results (crosses) are from Ref. [4]. Calculations are represented in solid (dashed) line for the soft EOS with (without) asymmetry term. The evaporative component with GEMINI simulation is in dotted-dashed line. (b) Differential cross sections in ${ }^{208} \mathrm{~Pb}(\mathrm{p}, \mathrm{Xp})$ reaction at $63 \mathrm{MeV}$. Experimental results (crosses) are from Ref. [4]. Theoretical calculations are drawn according to the previous conventions.

Let us examine the experimental differential cross sections for the ${ }^{208} \mathrm{~Pb}(\mathrm{p}, \mathrm{Xn})$ 
reaction at $63 \mathrm{MeV}$ shown on Fig. $2 \mathrm{a}$ and the ${ }^{208} \mathrm{~Pb}(\mathrm{p}, \mathrm{Xp})$ reaction at the same energy, displayed on Fig. 2b, both data extracted from Ref. [4]. As a matter of fact the pre-equilibrium component of the energy differential cross section of ${ }^{208} \mathrm{~Pb}(\mathrm{p}, \mathrm{Xn})$ channels are of the same order or higher than those of ${ }^{208} \mathrm{~Pb}(\mathrm{p}, \mathrm{Xp})$. The difference increases when one gets closer to the evaporative regime for the lowest emission energies, the Coulomb barrier of the lead target hindering the proton emission. In the present case the emission cross section are seemingly more in agreement with the magnitude sorting of free diffusion cross sections. Nevertheless, when one only considers the pre-equilibrium components beyond the influence of evaporation, the differences between experimental results on Fig. 2a and Fig. 2b are rather weak, this fact is also evidenced in the theoretical calculations. Despite the fact that we are investigating the out-of-equilibrium components of the differential cross section, let us briefly give some informations on the evoporative contibrutions due to the emission processes taking place after the stopping time of the dynamical simulation. As expected, in Fig. 2a one observes strong amplitudes for the emitted particles at low energies. In Fig. 2b, on contrary, the evaporative contribution to the spectrum is rather small. Estimations given by the GEMINI simulations provide the contribution which lacks to retreive the experimental magnitudes at the lowest energies.

As already stated, the theoretical results are given by solid (dashed) lines when they stem from a Skyrme force with (without) the isospin symmetry bulk contribution. The theoretical results provide reasonable estimates of the experimental data, with nevertheless an overestimation for the higher emission energies mainly in the $\mathrm{Pb}(\mathrm{p}, \mathrm{Xn})$ channel (Fig. 2a). As it has been underlined in ref. [18], the current DYWAN simulations deliberately rely on residual interactions driven by the free nucleon-nucleon cross section, including the observed energy and angular dependences. In medium effects [23] are expected to weaken the nucleon-nucleon cross section. The absence of this effects implies an overestimation of the spectra, especially for the higher emission energies.

Among the in medium effects, those due to the non-locality of the effective interaction are of special interest and have consequences on numerous observables [24]. Different effects can cooperate or compete in the cross section amplitudes. As a matter of fact the related momentum dependence introduces a more repulsive interaction at high relative momenta between the colliding partners. In connection, the induced nucleon effective masses will decrease the transition rates between the energy levels and could therefore affect the emission spectra principally at their high energy tails. It can be underlined that the model has been designed in order to incorporate more realistic forces, especially non-local interactions like the Gogny force 
and, consequently, it is able to cure a preponderant contribution to the observed discrepancies in future calculations.
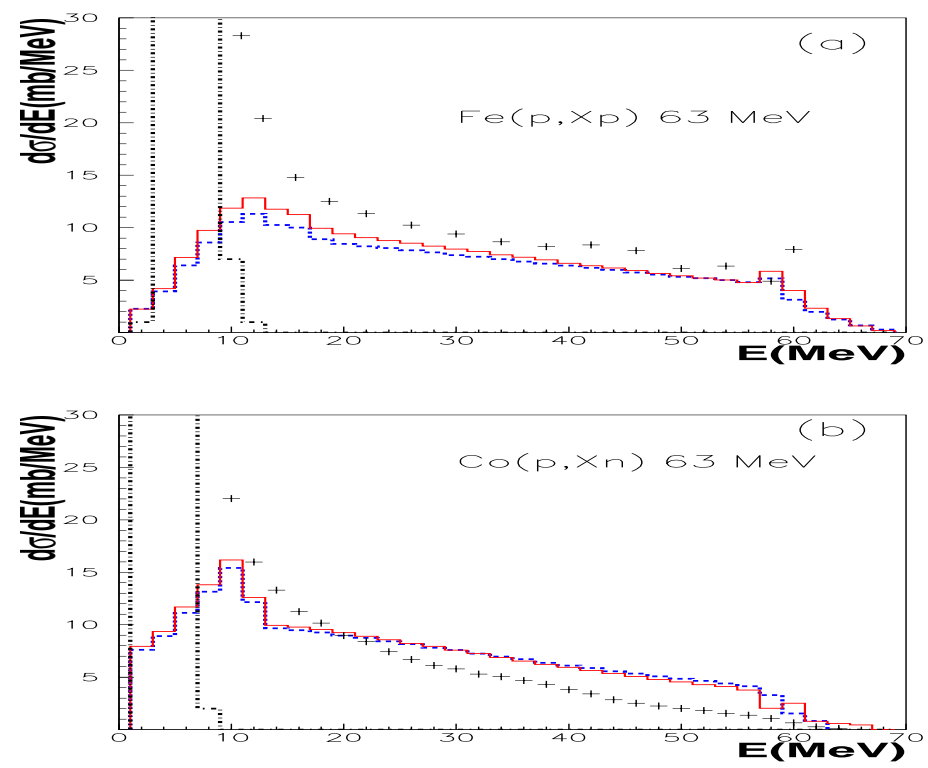

Figure 3: (Color online) Differential cross sections in ${ }^{56} \mathrm{Fe}(\mathrm{p}, \mathrm{Xp})(\mathrm{a})$ and ${ }^{59} \mathrm{Co}(\mathrm{p}, \mathrm{Xn})$ (b) reactions at $63 \mathrm{MeV}$. Experimental results are from Ref. [5]. Theoretical calculations are represented with solid (dashed) line for the soft EOS with (without) the asymmetry term. The evaporative component with GEMINI simulation is in dotted-dashed line.

Since the differential cross section in Fig. 2b exhibits a conspicuous sensitivity to the bulk isospin asymmetry of the effective interaction, to complete the investigation on the energy cross section of emitted nucleons, let us turn now to the emission processes related to lighter targets, in order to analyze the influence of the size of the target. This is the purpose of Fig. 3 where are displayed the results for the ${ }^{59} \mathrm{Co}(\mathrm{p}, \mathrm{Xn})$ and ${ }^{56} \mathrm{Fe}(\mathrm{p}, \mathrm{Xp})$ channels. The ${ }^{56} \mathrm{Fe}(\mathrm{p}, \mathrm{Xp})$ experimental data are extracted from Ref. [5] and those related to ${ }^{59} \mathrm{Co}(\mathrm{p}, \mathrm{Xn})$ are accessible on nuclear data files [25]. DYWAN results are represented using the conventions of figures $2 \mathrm{a}$ and 2b. In Fig. 3 a striking property is put forward by the theoretical results: contrary to what was shown in Fig. $2 \mathrm{~b}$ the sensitivity to the isospin asymmetry of the nuclear interaction is now washed out. This can be interpreted as a mass effect. Indeed, the fingerprints of the isospin asymmetry in calculations are expected to vanish since the net neutron-to-proton asymmetry decreases as the nuclei become lighter. 
The overestimation of the theoretical results in the $(\mathrm{p}, \mathrm{Xn})$ channel already observed for the higher energies in Fig. 2a is also present in Fig. 3b. As already pointed out, besides the influence of the simplified force, an important aspect is the deliberate choice to drive the residual interaction effects in accordance with the free nucleonnucleon cross section. In addition, it must be recalled that the lack of in-medium effects in the current treatment of the residual interactions leads to an underestimation of the spectra at forward angles and to an overestimation in the transverse direction. This effect is hardly detected in single differential cross sections. For this reason the information contained in Fig. 3a is somewhat degenerated and the apparent good agreement between experimental and theoretical results must not hide this aspect, which will be also addressed later on. Despite the lack of experimental simple differential cross sections for the ${ }^{56} \mathrm{Fe}(\mathrm{n}, \mathrm{Xp})$ reactions in the vicinity of 60 $\mathrm{MeV}$, those of ref. [3] can be extrapolated and should infer that cross sections for ${ }^{56} \mathrm{Fe}(\mathrm{n}, \mathrm{Xp})$ and ${ }^{56} \mathrm{Fe}(\mathrm{p}, \mathrm{Xn})$ are very similar.

\section{Emission cross section hierarchy}

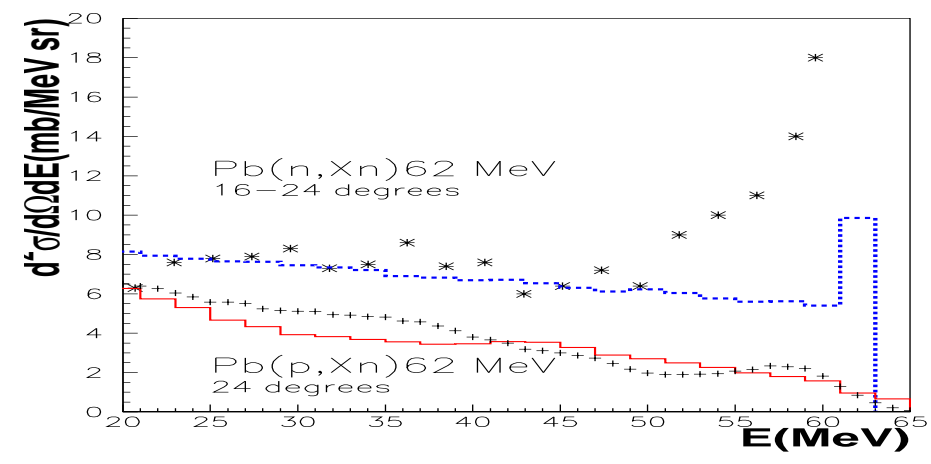

Figure 4: (Color online) Double-differential cross section in ${ }^{208} \mathrm{~Pb}(\mathrm{n}, \mathrm{Xn})$ reaction at $62 \mathrm{MeV}$ : theoretical values (dotted line) are compared with the experimental results from Ref. [26]. DYWAN results include a bulk isospin asymmetry term in the local Skyrme effective interaction. Double-differential cross section in ${ }^{208} \mathrm{~Pb}(\mathrm{p}, \mathrm{Xn})$ reaction at $62 \mathrm{MeV}$ : theoretical values (solid line) are compared with the experimental results from Ref. [4].

The Previous comparative investigation between theoretical and experimental results shows that a hierarchy of emission cross sections emerges for the heavier tar- 


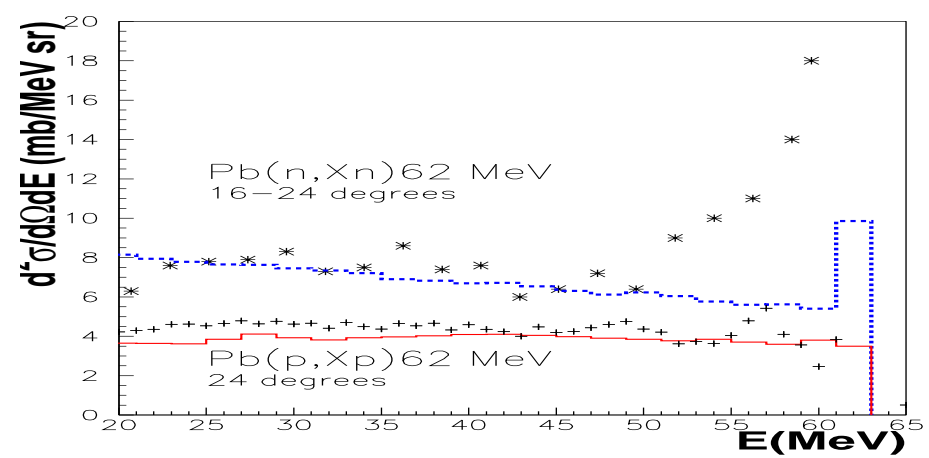

Figure 5: (Color online) Double-differential cross section in ${ }^{208} \mathrm{~Pb}(\mathrm{n}, \mathrm{Xn})$ [26] and in ${ }^{208} \mathrm{~Pb}(\mathrm{p}, \mathrm{Xp})$ [4] reactions at $62 \mathrm{MeV}$. Theoretical values include a bulk isospin asymmetry term in the local Skyrme effective interaction. In solid line are represented the calculations for the ${ }^{208} \mathrm{~Pb}(\mathrm{p}, \mathrm{Xp})$ channel and in dotted line those for the ${ }^{208} \mathrm{~Pb}(\mathrm{n}, \mathrm{Xn})$ one.

gets, namely the lead target. As a matter of fact, cross section magnitudes increase along with the following reactions: ${ }^{208} \mathrm{~Pb}(\mathrm{n}, \mathrm{Xp}),{ }^{208} \mathrm{~Pb}(\mathrm{p}, \mathrm{Xp})$ and ${ }^{208} \mathrm{~Pb}(\mathrm{p}, \mathrm{Xn})$. Let us now address the comparison of the energy cross sections in the ${ }^{208} \mathrm{~Pb}(\mathrm{p}, \mathrm{Xn})$ and ${ }^{208} \mathrm{~Pb}(\mathrm{n}, \mathrm{Xn})$ channels. The experimental data are relatively rare, and are often restricted to double differential cross sections of emitted particles for a small number of angles. Simple differential cross sections are therefore reconstructed using empirical laws, which renders the dubbed experimental results somewhat, model dependent, especially when a small number of angles are included in the systematics. Owing to this fact, it has been chosen here to resort directly to the experimental double differential cross sections which cannot be suspected to have been biased by any model. Afterwards, we will present results for only one angle, nevertheless it has been checked that the related trends remain true for other known experimental data at other angles.

In Fig. 4 are displayed the DYWAN results using the isospin dependent local Skyrme effective interaction in comparison with the experimental data from Ref. [26] in what concerns the ${ }^{208} \mathrm{~Pb}(\mathrm{n}, \mathrm{Xn})$ channels. We will afterwards use only this implementation of the local Skyrme effective interaction. The experimental results related to ${ }^{208} \mathrm{~Pb}(\mathrm{p}, \mathrm{Xn})$ reactions are extracted from Ref. [4]. In order to focus on the pre-equilibrium components, we consider only emitted nucleons with energies above $20 \mathrm{MeV}$, disregarding the evaporative contributions as well as eventual biases 
due to experimental thresholds of detection [26]. As in Fig. 1, one remarks again that the emission probability is higher when the projectile and the emitted nucleon are the same particle. This behaviour was interpreted as a pure mean-field effect. The essential role of these effects are therefore confronted through the emission characteristics of either protons in Fig. 1c or neutrons in Fig. 4. Let us remark that the results of Fig. 4 correspond to scattering angles around $25^{\circ}$. In References [4], [27] and [28] are shown the experimental double differential cross sections of emitted neutrons at $60^{\circ}$ for the reactions: ${ }^{208} \mathrm{~Pb}(\mathrm{p}, \mathrm{Xn})$ at $63 \mathrm{MeV},{ }^{208} \mathrm{~Pb}(\mathrm{p}, \mathrm{Xn})$ at $113 \mathrm{MeV}$ and ${ }^{208} \mathrm{~Pb}(\mathrm{n}, \mathrm{Xn})$ at $96 \mathrm{MeV}$. These experimental results reflect the same trends as Fig. 4: the strength of neutron spectra in neutron induced reactions is higher than in the case of incident protons, whatever the energy is.

In Fig. 2a and Fig. 2b, single differential cross sections in ${ }^{208} \mathrm{~Pb}(\mathrm{p}, \mathrm{Xn})$ and ${ }^{208} \mathrm{~Pb}(\mathrm{p}, \mathrm{Xp})$ at $63 \mathrm{MeV}$ incident energy have been discussed. We would like to reexamine the differences between these two reactions, this time by considering their corresponding double differential cross sections. We will then compare the yields of ${ }^{208} \mathrm{~Pb}(\mathrm{p}, \mathrm{Xn})$ in Fig. 4 with those corresponding to the ${ }^{208} \mathrm{~Pb}(\mathrm{p}, \mathrm{Xp})$ channel in Fig. 5. In this last picture the theoretical results are displayed for an angle of $24^{\circ}$ and with the same conventions of Fig. 4. Both cross sections exhibit a linear behaviour, the average value is roughly the same in the pre-equilibrium emission energy range, but the slopes are different. Indeed, in the ${ }^{208} \mathrm{~Pb}(\mathrm{p}, \mathrm{Xn})$ case the slope is negative while in the ${ }^{208} \mathrm{~Pb}(\mathrm{p}, \mathrm{Xp})$ case it is nearly zero. If we compare now the results for the two reactions of Fig. 5 one observes, as expected fom the above discussions, that the yields of the ${ }^{208} \mathrm{~Pb}(\mathrm{n}, \mathrm{Xn})$ reaction are higher than the ${ }^{208} \mathrm{~Pb}(\mathrm{p}, \mathrm{Xp})$ ones and, in consequence, they are the absolute highest value. Therefore, in what concerns heavy targets, a clear hierarchy in the experimental and theoretical cross sections appears: according to increasing values of the differential cross sections they are organized as follows: ${ }^{208} \mathrm{~Pb}(\mathrm{n}, \mathrm{Xp}),{ }^{208} \mathrm{~Pb}(\mathrm{p}, \mathrm{Xp}),{ }^{208} \mathrm{~Pb}(\mathrm{p}, \mathrm{Xn})$ and ${ }^{208} \mathrm{~Pb}(\mathrm{n}, \mathrm{Xn})$.

To better evidence this hierarchy in a quantitative way, let us introduce a measure of the pre-equilibrium component of the differential cross section through its integrated values $\Lambda$, at a given angle, on an energy range of $30 \mathrm{MeV}$. Namely,

$$
\Lambda\left(E_{\min }, E_{\text {max }}\right)=\int_{E \min }^{\operatorname{Emax}} \frac{\mathrm{d}^{2} \sigma}{\mathrm{d} E \mathrm{~d} \Omega} d E,
$$

where $E_{\min }=20 \mathrm{MeV}$ and $E_{\max }=50 \mathrm{MeV}$ when the beam energy is around to 65 $\mathrm{MeV}$, and $E_{\text {min }}=40 \mathrm{MeV}$ and $E_{\max }=70 \mathrm{MeV}$ when the beam energy is close to $95 \mathrm{MeV}$. 

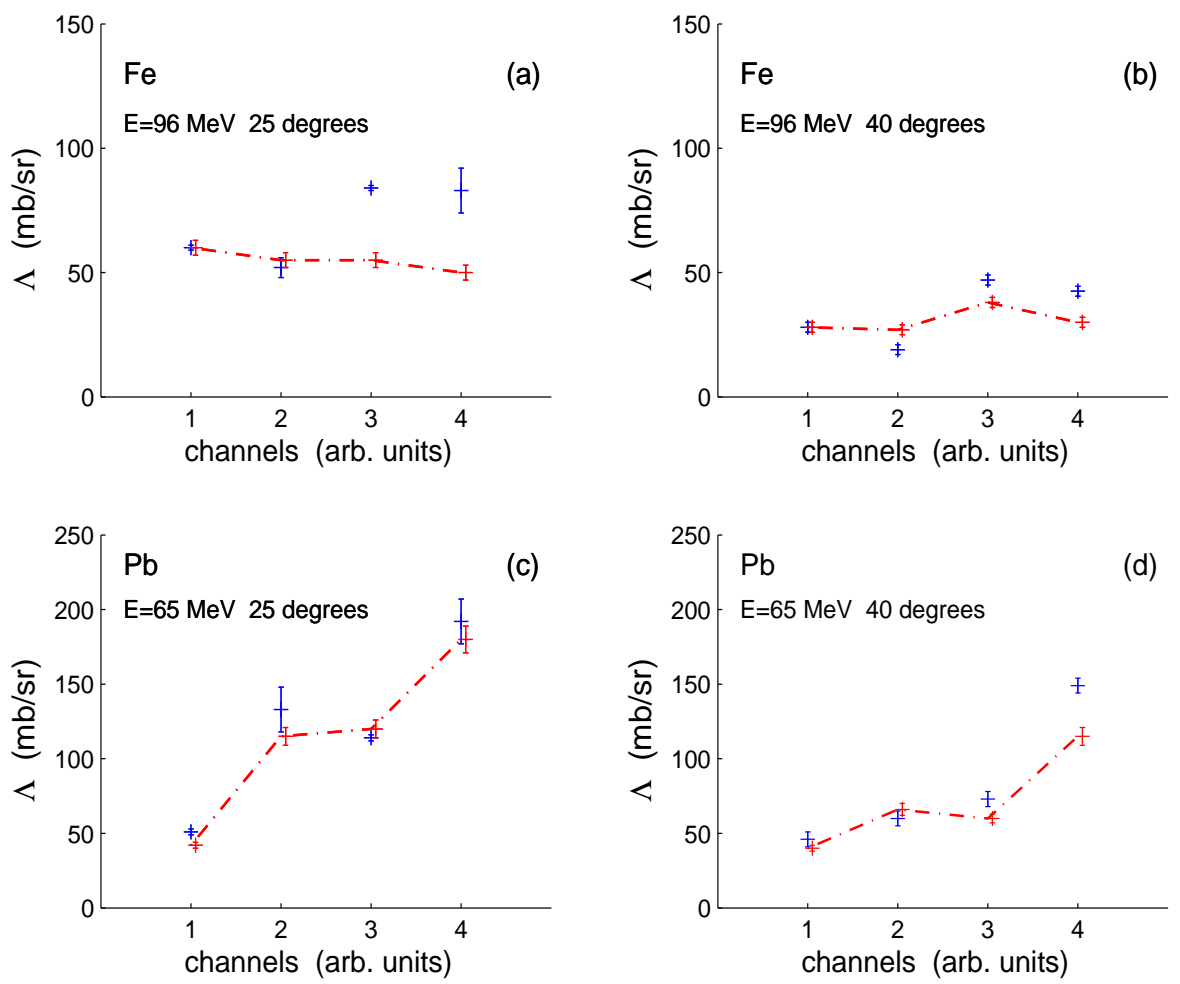

Figure 6: (Color online) Integrated differential cross sections on iron (top) and on lead (bottom) targets for two different angles and two incident energies. The results for different channels are reported. Points correspond to experimental results and the dashed line joins the theoretical values. See text for more details. 
A correlated purpose is also to investigate the relative sorting of the cross sections to the target masses. The results are plotted in Fig. 6, and the detailed numerical values are reported on Table 1. Two kinds of incident energies have been considered, respectively, around $65 \mathrm{MeV}$ and $95 \mathrm{MeV}$, for targets close to ${ }^{208} \mathrm{~Pb}$ and to ${ }^{56} \mathrm{Fe}$. In Fig. 6 the abscissa represents, in arbitrary units, the different channels, $1:(\mathrm{n}, \mathrm{Xp}), 2$ : $(\mathrm{p}, \mathrm{Xn}), 3:(\mathrm{p}, \mathrm{Xp})$ and $4:(\mathrm{n}, \mathrm{Xn})$. We observe that the experimental curves increase with the defined sorting. For the lighter targets, the theoretical curves are rather flat with a slight increase only at the highest energy. For these targets the theoretical results differ from the experimental ones mainly at the most forward angle. This effect is due to the lack of in-medium effects in the residual interactions, as for exemple the effective mass. As a matter of fact, the free nucleon-nucleon cross section is too high, causing the depopulation of the spectra at forward angles and, correspondingly, the overestimation in the transverse direction. For the heavy target the theoretical cross sections increase in the above defined sorting, exhibiting a plateau in the middle for all angles and energies in agreement with the measured values. These results evidence the importance of the mean-field in the case of heavy targets. Even more, from the discussion of Fig. 5 it is clearly more repulsively in the $(\mathrm{n}, \mathrm{Xn})$ channel than in the $(\mathrm{p}, \mathrm{Xp})$ one. Since we are studying beam energies between

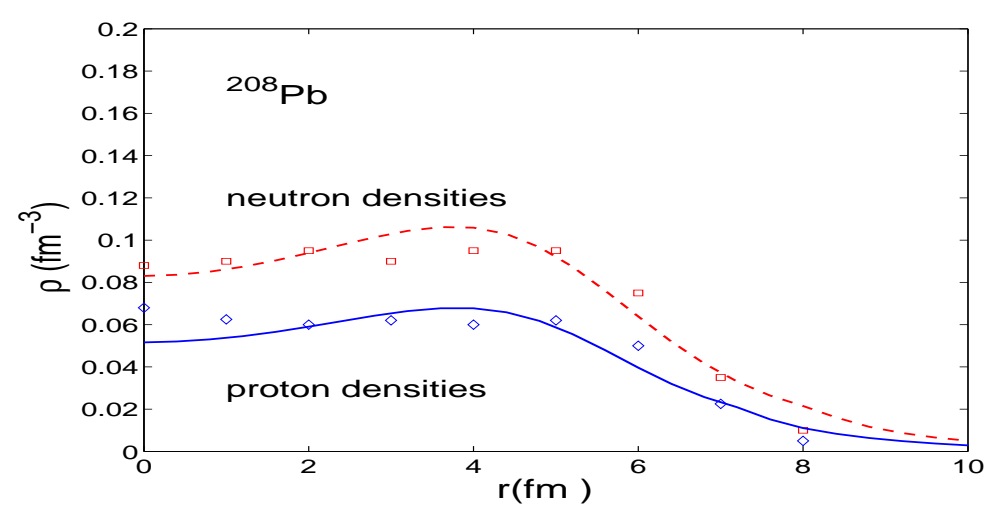

Figure 7: (Color online) Neutron (dashed line) and proton (solid line) density profiles calculated with the DYWAN approach. In diamonds and squares are the correponding results of Ref. [33].

$20 \mathrm{MeV}$ and $200 \mathrm{MeV}$, the nucleon-nucleus collisons are probing preferentially the nuclear characteristics of the target surface. Consequently the common experimental and theoretical behaviors strongly suggest the presence of neutron excess at the surface. In order to illustrate this result, in Fig. 7 we have represented separately 
the neutron and proton densities provided by the current statics of the DYWAN model which, in this framework, stems from the lowest level scale of approximation. Together with our calculations, in Fig. 7 are depicted in diamonds and squares those corresponding to Relativistic Mean field models [32, 33]. The results indicate that, at the concerned energies, nucleon-induced reactions favours the interaction of the incoming projectile mainly with the neutron skin.

\section{Conclusions}

The measured free nucleon-nucleon diffusions evidence that proton-neutron cross sections are higher than proton-proton and neutron-neutron ones. Cross sections provided by nucleon-induced experiments are higher in the ${ }^{208} \mathrm{~Pb}(\mathrm{p}, \mathrm{Xp})$ reaction than in the ${ }^{208} \mathrm{~Pb}(\mathrm{n}, \mathrm{Xp})$ one. This states that mean-field effects strongly compete with direct diffusions, and even they can become predominant. Theoretical simulations exhibit a strong sensitivity to the isospin asymmetry of the effective local Skyrme interaction in ${ }^{208} \mathrm{~Pb}(\mathrm{p}, \mathrm{Xp})$ reactions. It means that in $(\mathrm{p}, \mathrm{Xp})$ channels compared with $(n, X p)$ ones, on the average, the emitted protons stem from a greater number of diffusions, and therefore experience the nuclear mean field longer, becoming more sensitive to the nuclear interaction properties governing the mean field [34], especially to the isospin asymmetry.

Nucleon-induced experiments show that cross sections of emitted nucleons are rather similar in ${ }^{208} \mathrm{~Pb}(\mathrm{p}, \mathrm{Xn})$ and in ${ }^{208} \mathrm{~Pb}(\mathrm{p}, \mathrm{Xp})$ reactions. This indicates, in this case, that the contribution of the mean-field is at least of the same order as direct diffusions. The current theoretical simulations exhibit an overestimation of ${ }^{208} \mathrm{~Pb}(\mathrm{p}, \mathrm{Xn})$ spectra for the higher emission energy. This is essentially due to the overestimated residual interactions and to the implemented local interaction. Nevertheless, related in-medium effects and a more realistic effective interaction should be accessible in forthcoming refined and quantitative investigations.

Finally nucleon-induced experiments emphasize that the emitted nucleon cross sections are clearly higher in ${ }^{208} \mathrm{~Pb}(\mathrm{n}, \mathrm{Xn})$ than in ${ }^{208} \mathrm{~Pb}(\mathrm{p}, \mathrm{Xp})$ reactions. Since, the effect of the residual interactions are expected to be the same in both cases the previous result shows that the mean field acts more repulsively in the $(\mathrm{n}, \mathrm{Xn})$ channel than in the $(\mathrm{p}, \mathrm{Xp})$ one. From here we can infer the presence of a neutron skin for the heavy target. This is coherent with the fact that the energy involved in these nucleon-induced reactions favours the interaction of the incoming projectile mainly with the nuclear matter located at the surface of the target. 


\begin{tabular}{|c|c|c|c|c|}
\hline$\Lambda$ & $\mathrm{Pb}(\mathrm{n}, \mathrm{Xp})$ & $\mathrm{Pb}(\mathrm{n}, \mathrm{Xn})$ & $\mathrm{Pb}(\mathrm{p}, \mathrm{Xn})$ & $\mathrm{Pb}(\mathrm{p}, \mathrm{Xp})$ \\
\hline & $62.7 \mathrm{MeV}[2]$ & $65 \mathrm{MeV}[26]$ & $62.7 \mathrm{MeV}[4]$ & $62.7 \mathrm{MeV}[4]$ \\
\hline experimental & $30^{\circ}$ & $28^{\circ}$ & $24^{\circ}$ & $30^{\circ}$ \\
\hline$(\mathrm{mb} / \mathrm{sr})$ & $51 . \pm 2$ & $192 . \pm 15$ & $133 . \pm 15$ & $114 . \pm 2$ \\
\hline theoretical & $30^{\circ}$ & $25^{\circ}$ & $25^{\circ}$ & $25^{\circ}$ \\
\hline$(\mathrm{mb} / \mathrm{sr})$ & $42 . \pm 2$ & $180 . \pm 2$ & $115 . \pm 6$ & $120 . \pm 6$ \\
\hline$\Lambda$ & $\mathrm{Pb}(\mathrm{n}, \mathrm{Xp})$ & $\mathrm{Pb}(\mathrm{n}, \mathrm{Xn})$ & $\operatorname{Bi}(p, X n)$ & $\mathrm{Pb}(\mathrm{p}, \mathrm{Xp})$ \\
\hline & $96 \mathrm{MeV}[3]$ & $96 \mathrm{MeV}$ [28] & $90 \mathrm{MeV}$ [29] & $96 \mathrm{MeV}[30]$ \\
\hline experimental & $40^{\circ}$ & $40^{\circ}$ & $40^{\circ}$ & $40^{\circ}$ \\
\hline$(\mathrm{mb} / \mathrm{sr})$ & $46 . \pm 5$. & $149 . \pm 5$ & $60 . \pm 5$ & $73 . \pm 5$ \\
\hline theoretical & $40^{\circ}$ & $40^{\circ}$ & $40^{\circ}$ & $40^{\circ}$ \\
\hline$(\mathrm{mb} / \mathrm{sr})$ & $40 . \pm 2$ & $115 . \pm 6$. & $66 . \pm 4$. & $60 . \pm 3$ \\
\hline$\Lambda$ & $\mathrm{Co}(\mathrm{n}, \mathrm{Xp})$ & $\mathrm{Fe}(\mathrm{n}, \mathrm{Xn})$ & $\mathrm{Co}(\mathrm{p}, \mathrm{Xn})$ & $\mathrm{Fe}(\mathrm{p}, \mathrm{Xp})$ \\
\hline & $65 \mathrm{MeV}$ [31] & $65 \mathrm{MeV}$ [26] & $62 \mathrm{MeV}[25]$ & $61 \mathrm{MeV}[5]$ \\
\hline experimental & $30^{\circ}$ & $28^{\circ}$ & $24^{\circ}$ & $30^{\circ}$ \\
\hline & $53 . \pm 1$ & $80 . \pm 9$ & $50 . \pm 4$ & $75 . \pm 1$ \\
\hline theoretical & $25^{\circ}$ & $25^{\circ}$ & $25^{\circ}$ & $30^{\circ}$ \\
\hline$(\mathrm{mb} / \mathrm{sr})$ & $65 . \pm 3$ & $65 . \pm 3$ & $55 . \pm 3$ & $55 . \pm 3$ \\
\hline$\Lambda$ & $\mathrm{Fe}(\mathrm{n}, \mathrm{Xp})$ & $\mathrm{Fe}(\mathrm{n}, \mathrm{Xn})$ & $\mathrm{Ni}(\mathrm{p}, \mathrm{Xn})$ & $\mathrm{Cu}(\mathrm{p}, \mathrm{Xp})$ \\
\hline & $96 \mathrm{MeV}$ [3] & $96 \mathrm{MeV}[28]$ & $90 \mathrm{MeV}[29]$ & $96 \mathrm{MeV}[30]$ \\
\hline experimental & $40^{\circ}$ & $40^{\circ}$ & $40^{\circ}$ & $40^{\circ}$ \\
\hline & $28 . \pm 2$. & $42.5 \pm 2$. & $19 . \pm 2$. & $47 . \pm 2$. \\
\hline theoretical & $40^{\circ}$ & $40^{\circ}$ & $40^{\circ}$ & $40^{\circ}$ \\
\hline$(\mathrm{mb} / \mathrm{sr})$ & $28 . \pm 2$. & $30 . \pm 2$. & $27 . \pm 2$. & $38 . \pm 2$. \\
\hline
\end{tabular}

Table 1: Experimental and theoretical differential cross sections for various targets and different pairs of incoming-outgoing particles 
All these coherent and convergent experimental as well as theoretical indications strongly suggest that nucleon-induced reactions in the Fermi energy domain are valuable probes of the isospin asymmetry of both the nuclear matter in exotic nuclei and of the nuclear interaction. The current investigation points out that refinement in experiments and theoretical simulation could provide the opportunity to extract stringent and quantitative informations about the characteristics of the nuclear force.

\section{References}

[1] R.E. Segel et al ,Phys. Rev. C 26 (1982) 2424; W. Scobel et al., ibid. C 41 (1990) 2010; J.R.Wu, C.C. Chang and H.D. Holmgren, ibid. C 19 (1979) 698; HINDAS, FIKW-CT-2000-0031, in: J.P. Meulders, A. Koning, S. Leray (editors), European Commission Contract, Final Report, 2005.

[2] M. Kerveno et al., Phys. Rev. C 66 (2002) 014601.

[3] V. Blideanu et al., Phys. Rev. C 70 (2004)014607.

[4] A. Guertin et al, Eur. Phys. J. A 23 (2005)49.

[5] F. E. Bertrand, R. W. Peelle, Phys. Rev. C 81045 (1973).

[6] J. J. Griffin, Phys. Lett. 17 (1966) 478; I. Ribanský and P. Obložinský, Phys. Lett. B 45 (1973) 318; J. R. Wu and C. C. Chang, Phys. Rev. C 17 (1978) 1540; C. Kalbach and F.M. Mann, ibid. 23 (1981) 112; A. J. Koning and J. P. Delaroche, Nucl. Phys. A 713 (2003) 231; J. Cugnon and P. Henrotte Eur. Phys. J. A 16 (2003) 393; D. Lacroix, V. Blideanu and D. Durand Phys. Rev. C 71 (2005) 024601.

[7] F. Sébille, C. Bonilla, V. Blideanu and J.F. Lecolley, Nucl. Phys. A 756 (2005) 229.

[8] V. de la Mota and F. Sébille, Eur. Phys. J. A 12 (2001) 479.

[9] S. Mallat, A Wavelet Tour of Signal Processing (Academic Press, New York 1999); I. Daubechies Ten Lectures on Wavelets (SIAM, Philadelphia, 1992).

[10] B. Jouault, F. Sébille and V. de la Mota, Nucl.Phys. A 628 (1998) 119. 
[11] R. J. Charity et al., Nucl. Phys. A 483 (1988) 371, 511 (1990) 59.

[12] F. Sébille, V. de la Mota, I.C. Sagrado Garcia, J.F. Lecolley and V. Blideanu, Nucl. Phys. A 791 (2007) 313 .

[13] M.B. Tsang et al., Phys. Rev. Lett. 92 (2004) 062701.

[14] Bao-An Li, Phys. Rev. C 69 (2004) 034614.

[15] S.T. Ali, J.P. Antoine, J.P. Gazeau, U.A. Mueller, Rev. Math. Phys.7 (1995) 1013.

[16] A. Ono, P. Danielewicz, W.A. Friedman, W.G. Lynch and M.B. Tsang, Phys. Rev. C 68 (2003) 051601(R).

[17] M. Unser, in Wavelets: A Tutorial in Theory, edited by C.K. Chui (Academic Press, New York, 1992).

[18] F. Sébille, C. Bonilla, V. Blideanu and J.F. Lecolley, Nucl. Phys. A 756 (2005) 229.

[19] L. Zamick, Phys. Lett. B 45 (1973) 313.

[20] M. Farine, T. Sami, B. Remaud and F. Sébille, Z. Phys. A 339 (1991) 363.

[21] B.G. Harvey, J. Phys. 47 (1986) c4-29.

[22] J. Binstock, Phys. Rev. C 10 (1974) 19.

[23] G.Q. Li, R. Machleidt, Phys. Rev. C 48 (1993) 1702; G.Q. Li, R. Machleidt, Phys. Rev. C 49 (1994) 566.

[24] F. Haddad, B. Borderie, V. de la Mota, M.F. Rivet, F. Sébille and B. Jouault, Z. Phys. A 354 (1996) 321.

[25] EXFOR Nuclear Data, Nuclear Energy Agency.

[26] E.L. Hjort et al., Phys. Rev. C 53 (1996) 237.

[27] M.M. Meier et al, Nucl. Sci. Eng. 102 (1989) 310.

[28] I.C. Sagrado Garcia, Ph.D. Thesis, Caen University (France) 2006.

[29] A.M.Kalend et al., Phys. Rev. C 28 (1983) 105.

[30] K. Strauch and F.Titus, Phys. Rev. 104 (1956) 191. 
[31] N. Nica, S. Benck, E. Raeymackers, I.Slypen, J.P.Meulders and V.Corcalciuc, J. Phys. G 28 (2002) 2823.

[32] J. Piekarewicz, S.P. Weppner; Nucl. Phys. A 778 (2006) 10.

[33] B.G. Todd, J. Piekarewicz, Phys. Rev. C 67 (2003) 044317.

[34] B. Jouault, V. de la Mota, F. Sébille, G. Royer and J.F. Lecolley; Nucl. Phys. A 597 (1996) 136. 